\title{
Design and Analysis of Mobile Learning Management System based on Web App
}

\author{
Shinwon Lee \\ Department of Computer System Engineering, \\ Jungwon University, Chungbuk, Republic of Korea \\ swlee@jwu.ac.kr
}

\begin{abstract}
We can take the e-Learning using smart devices on information society is that wellformed internet environment. LMS (Learning Management System) supports mobile video lectures. The proposed system designs that instructors can set up lectures without the professional computer related technologies, and the interaction between instructors and learners becomes easy. This system focuses on helping the interaction between instructors and learners made easier by using a web browser in the mobile device based on web app, anytime and anywhere learners want to use the system.
\end{abstract}

Keywords: Learning Management System, Mobile, Video, Web App

\section{Introduction}

With the development of information and communication technology, which is also known as the internet revolution, internet-based information age is coming and e-Learning technology is also developed. It is possible that we can learn using the internet as the advancement of smart devices. Smart environment is mobile ability of existing information devices, and cloud computing is that the storage and process of information are mass. It is possible that the mobility has unimaginable usage with PC-based and the cloud computing develops intelligent technology with mass information management and processing. So smart learning is a new paradigm for elearning through the use of smart devices and intelligent technologies in a smart environment. The contents, solution, and the service based on PC have problems in adapting into a mobile environment. However, smart learning is possible to implement in a smart environment $[7,8]$.

It needs the technology to change them to the mobile environment that the contents, solution, service are implemented through traditional PC-base programs. The environment of Web 2.0 is developing to participation data of users and is influencing to the learning environment of web. We can use contents of Web 2.0 on learning, but educational contents made using this technology especially for domestic video contents are served through not open API, such as Adobe's Flash Player. Such a commercially available educational support system is large and increases cost accordingly because it includes all of the conditions that occur in an individual study area due to custom development $[1,2]$.

The proposed system has opened the lecture for instructors and students through a simple process, but it does not depend on a particular vendor or lecture places. It saves management cost of the video server for maximizing the training effect, and supports learners to achieve the maximization of learning with a community of users.

In this paper, without the use of a commercially available system, we design the simple mobile learning management system that is supported by video clips to help instructors and learner study more efficiently. 


\section{Related Research}

The existing learning method using the internet has the disadvantage that the interaction between instructors and learners progresses one-way only, it does not provide a practical learning environment for learners [12].

As an alternative to overcome these drawbacks, the advantage of video data in the class can be described as causing the interest of learners and extended the limited training space outside of the classroom to overcome the educational limitations [13]. In addition, recently the emergence of a social network service may be a solution to overcome the difficulty of the interaction in the e-learning environment [11]. However, the problems with the method using the normal video data are an increase of traffic and copyright. The copyright issue is not a problem because the actor to serve is instructor.

The manager to operate the system has the biggest problem that how it handles rapidly growing network traffic in order to reduce maintenance cost. It appears that the cost of network management raises [14].

This paper designs learning environment in order to reduce the cost using the video service and also reduce the difficulty of the interaction of the existing support system.

Learning management system (LMS) is a system that supports and manages all items in the learning environment of learners and all items in the teaching environment of instructors, and refers to a remote learning management system on web-based. Figure 1 is a general diagram of the learning management system [8, 10].

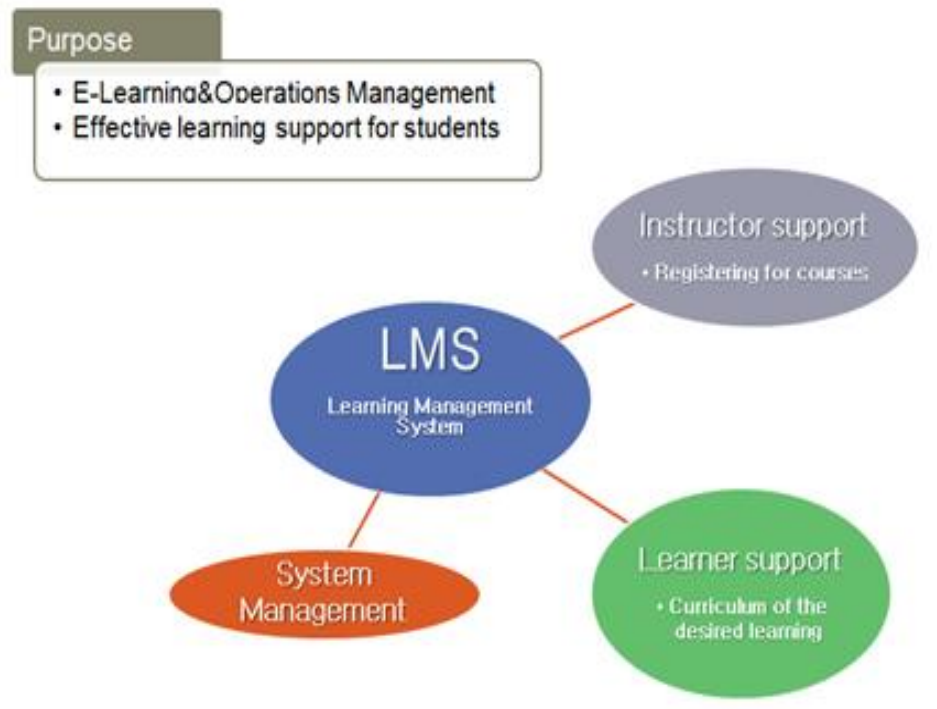

Figure 1. Diagram of Learning Management System

The manager has to prepare the learning system that is instructors and learners participate in the study such as opening the curriculum of the desired learning for learners, registering for courses in cyberspace. After preparation is completed, on the process of real learning, the system provides individual learners with tracing of learning progress, managing of learning history, and personalized learning.

The main features of LMS is class function, cooperative learning function, attendance management, board functions, etc. which are required for online learning. LMS needs the technology for processing video lectures and the cost that makes the video, store, and replay of the lectures $[7,9]$. 


\section{Proposed Method and System Design}

\subsection{System Configuration}

This system reduces the function which is not used primarily to existing learning support systems and adds the video service information of which the learners manage the curriculum on their own. Figure 2 is proposed method. This system suggests the function that can be done through the video communication between learners. The existing learning system develops the content for the learning object unit. This supports less or no multimedia content, and derives general interactive elements.

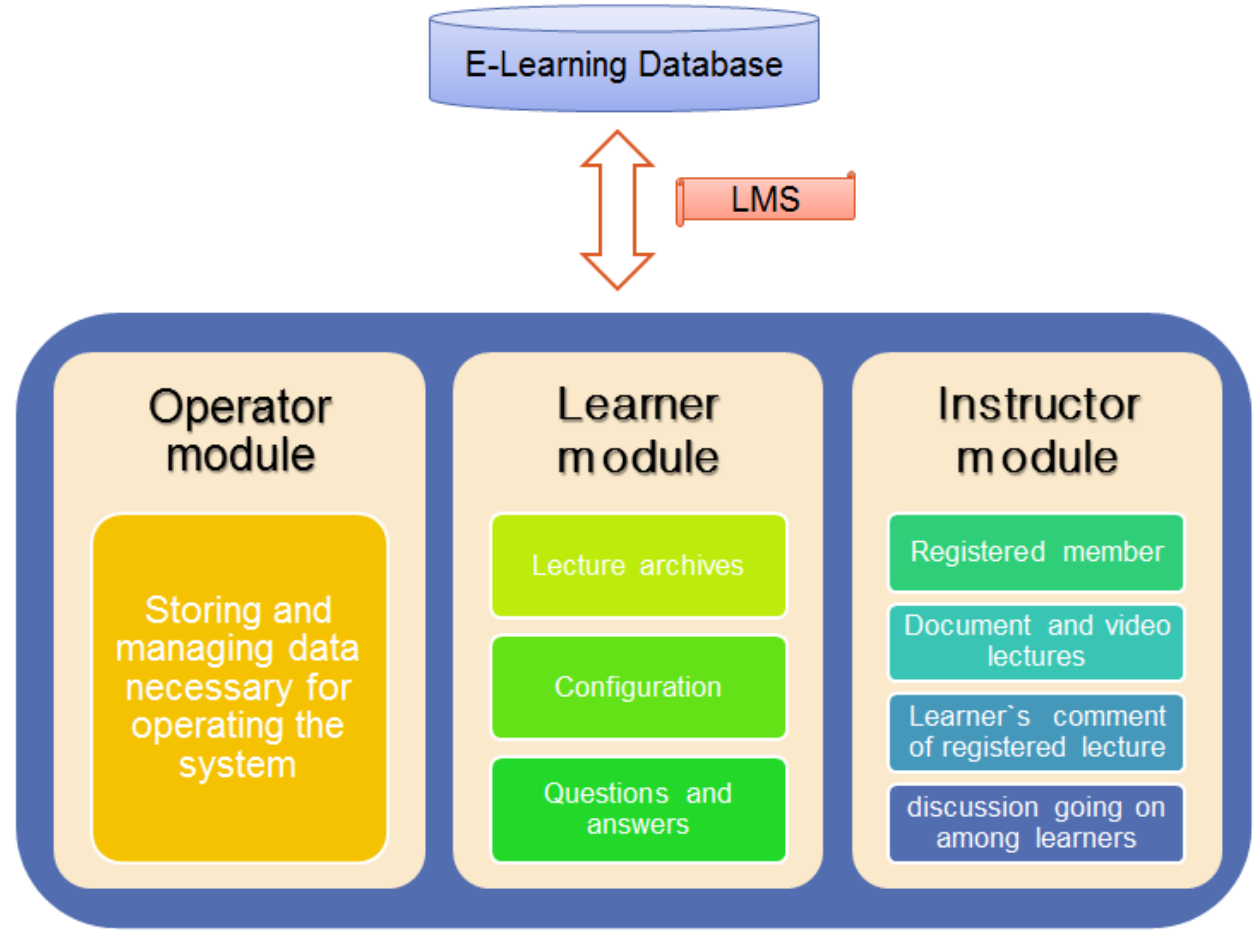

Figure 2. The Proposed Method

System components are as follows.

1) Database: used in conjunction with the data of learner module and instructor module

2) Operator module: module for which the operator is storing and managing data necessary for operating the system

3) Learner module: having a lecture archives, configuration, such as questions and answers

4) Instructor Module:

- The instructor registered member registers documents and video lectures

- The collecting through learner's comment of registered lecture

- The discussion going on among learners through lecture board 
E-learning is the system that anytime, anywhere, anyone can study to customized learning levels using information and communication technologies. This system is based on e-learning and designed to apply user-friendly and efficiently when instructors progress their lectures.

Figure 3 is the framework of proposed LMS.

The learner module consists of all lectures including new one and learner's lecture management including searching, implicating and participating. The learner can apply online lecture efficiently.

The Database of LMS is used in association with each other data of the learner module and instructor module.

In the instructor module, instructors can register documents and videos of lectures. And this has the feedback function that converge learner's comments, and students can discuss lectures through the board of students.

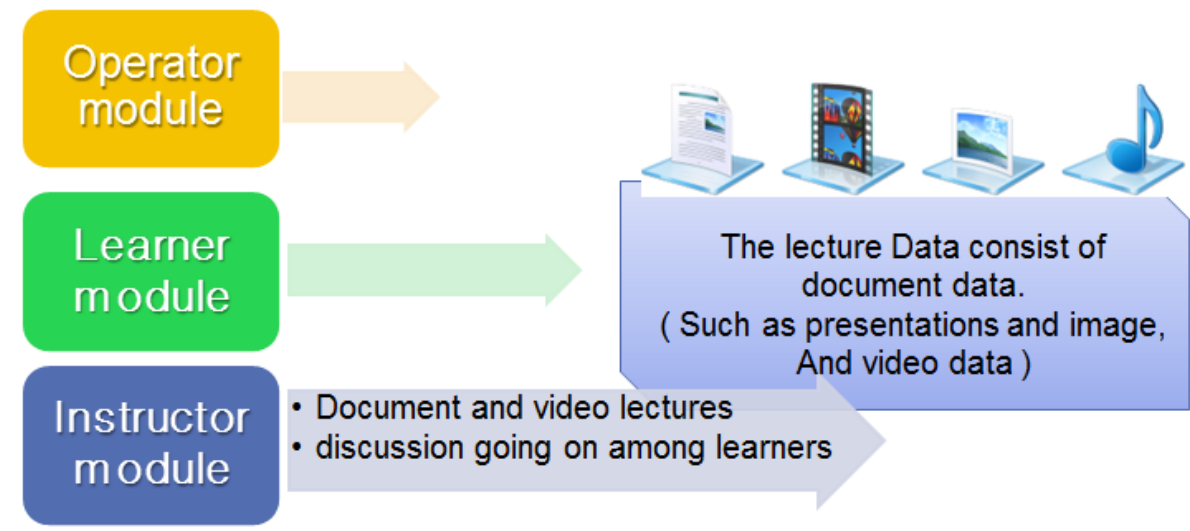

Figure 3. The Framework of Proposed LMS

The lecture data consist of document data with characters such as presentations, images, and video data that maximize visual effects. Video data is larger than characters and images. The seamless learning support is difficult due to the increase of use of server's hard disk and network traffic.

To solve this problem, we propose a way of "Browser-based upload" to upload via the YouTube API-based and video playback. Figure 4 and 5 show the upload process for pre-processing and image processing for using the API [3].

\section{API Requirements}

\section{Register to submit your application to the Google API requests. \\ ( https://developers.google.com/youtube/registering_an_application ) \\ 2. To grasp the concept of JSON (JavaScript Object Notation) data type.}

Building the application

1. Read the YouTube Data API Getting Started Guide. ( https://developers.google.com/youtube/v3/getting-started )

2. Please select the client implements the API library. ( https://developers.google.com/youtube/v3/libraries )

3. Understand how to implement OAuth 2.0 authentication.

Figure 4. Procedure for using the API 


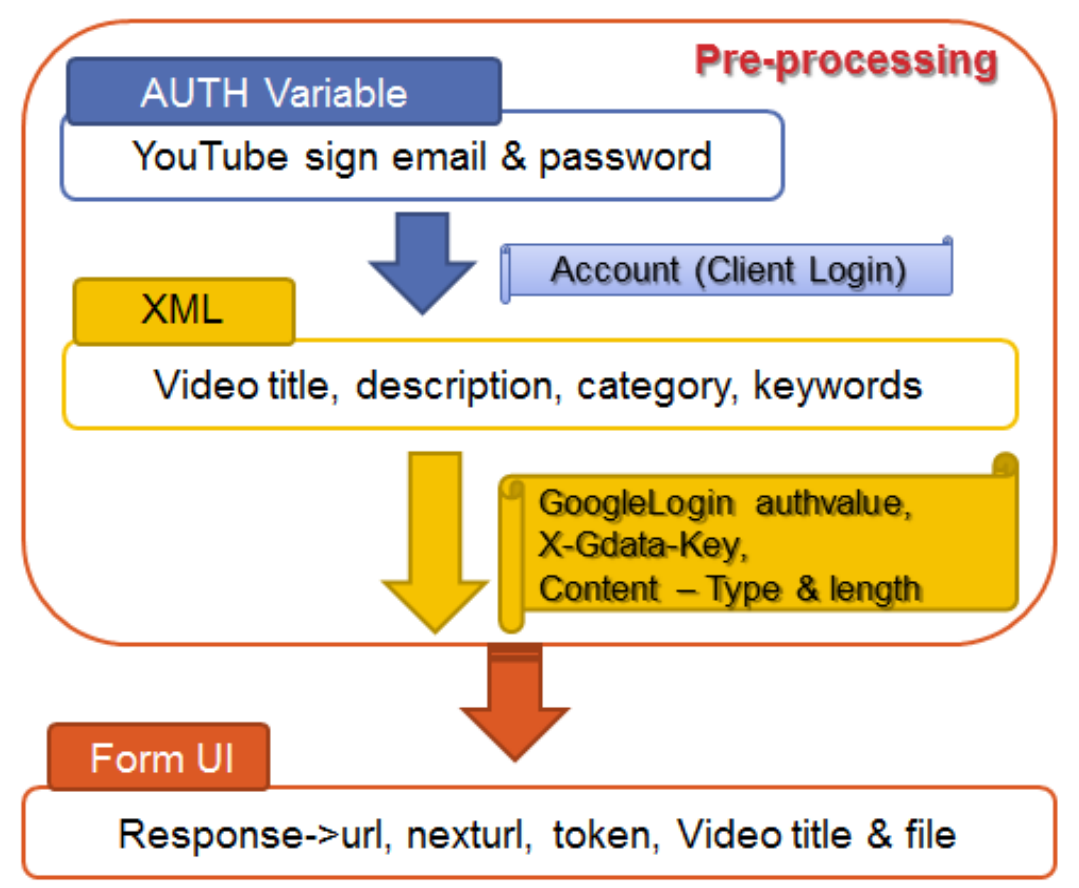

Figure 5. "Browser-based upload" with YouTube

Figure 6 is YouTube video manager page. Before applying this system, it tests with various options and checks the execution result of the sample moving images. The system was validated and applied to the video through the testing process.

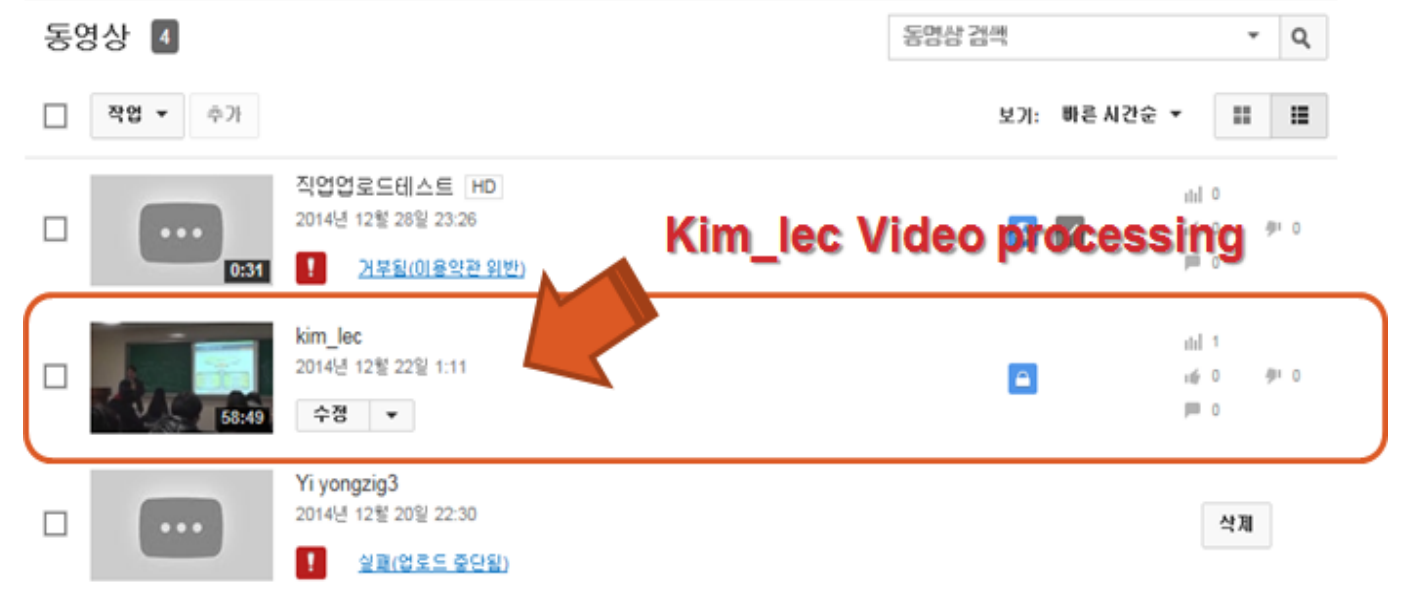

Figure 6. YouTube Video Manager Page

\subsection{The Online Video Service Method}

HTML (Hypertext Markup Language) is a basic programming language used to create web pages for online services. It is developed to create hypertext and most web pages that are accessible through the internet are written in HTML.

HTML is made to define the format of the electronic document and defined as a subset of the international standard SGML. HTML is a language created to emphasize in particular hypertext in SGML.

We usually use $<E M B E D>$ tag to insert video or music data online. How to use tags and attributes are shown in Table 1. 
<EMBED SRC="Filename" WIDTH="player width" HEIGHT="player height"

AUTOSTART="true | false" LOOP="true | false |loopcount" HIDDEN="true | false" $>$

Table 1. EMBED Methods and Properties

\begin{tabular}{l|l}
\hline \multicolumn{1}{c|}{ Attribute } & \multicolumn{1}{c}{ Description } \\
\hline SRC="Filename" & Location of the music or video files with the file name. \\
\hline WIDTH="player width" & Defines the width of the player. \\
\hline HEIGHT=" player height" & Defines the height of the player. \\
\hline AUTOSTART="true|false" & Open the document defines whether playing music or movies. \\
\hline LOOP="true|false|loopcount" & Defines whether or not to repeat a few times. \\
\hline HIDDEN="true|false" & Defines whether to hide the player on the screen. \\
\hline
\end{tabular}

This system plays Table 2's image using above tag via a web browser and applies the data traffic as shown in Figure 7.

Table 2. Specification of a Sample Video

\begin{tabular}{c|c|c|c}
\hline File Name & Size (KB) & Resolution & Duration \\
\hline Kim_lec.wmv & $3,710,842,723$ & $1440 \times 1080$ & $00: 58: 48$ \\
\hline
\end{tabular}

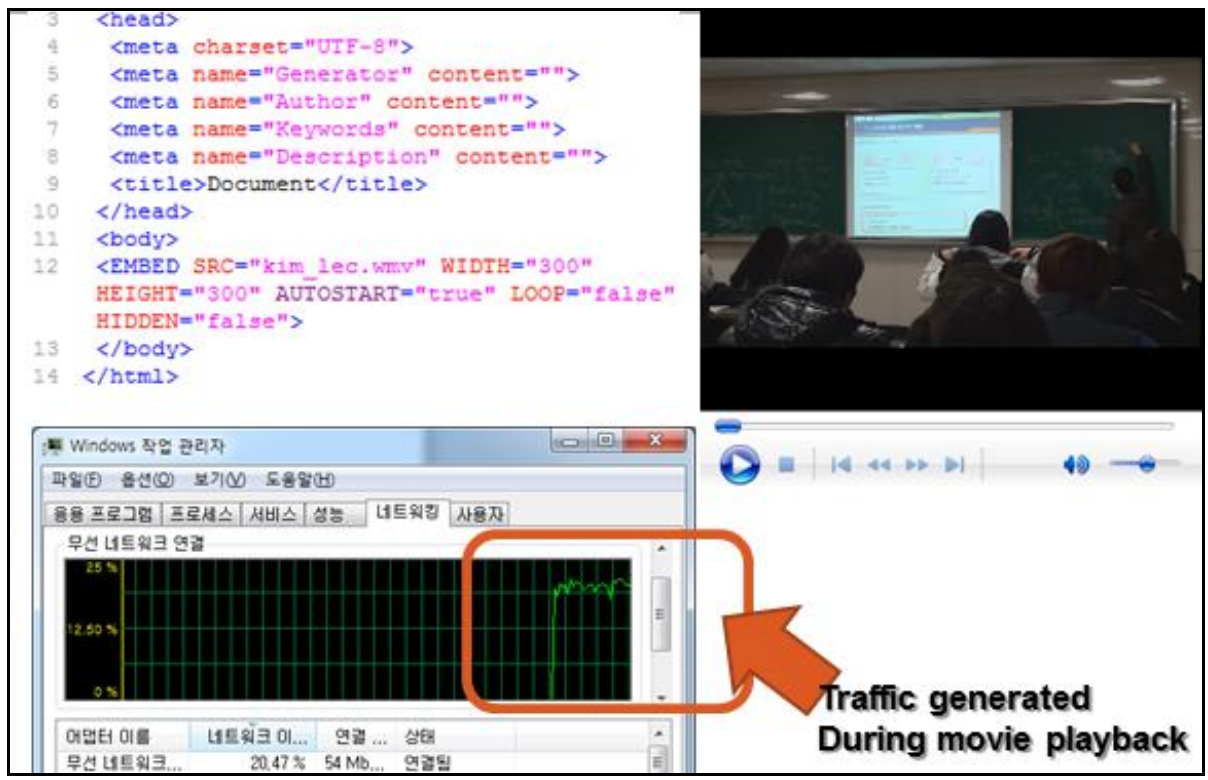

Figure 7. Playing of video and graph of the traffic data

This service can operate to save the image data to the server specified by the learning system. But it needs preparation process for image service and occur additional maintenance and service costs.

The process for video service has a sequence as shown below.

1) Make lecture video 
2) Save and edit captured images

3) Encode video for video services and determine the optimal image quality

4) Upload to the video storage server and associated address (or HTML code) acquisition

5) Enter the acquired code in learning management system

6) Start the service

Problem of the service maintenance costs beyond the complicated process for preparing the above service (traffic) is to be generated. Briefly looking at the graphic of Figure 7 can check the network usage rate of about $22-25 \%$. For more information on the traffic and the image will be covering in detail in the "image selection and traffic analysis, optimized for 3.3 online". In this paper, a learning system solution to these problems, we propose a service applying an external video service YouTube. The video playback and the traffic graphs with YouTube are shown in Figure 8.

The process for the YouTube video service has a sequence as shown below.

1) Make lecture video

2) Save and edit captured images

3) (YouTube automatically determined) Encode video for video services and determine the optimal image quality

4) (Upload to YouTube UI soon) address (or HTML code) provided

5) Enter the acquired code in learning management system

6) Start the service

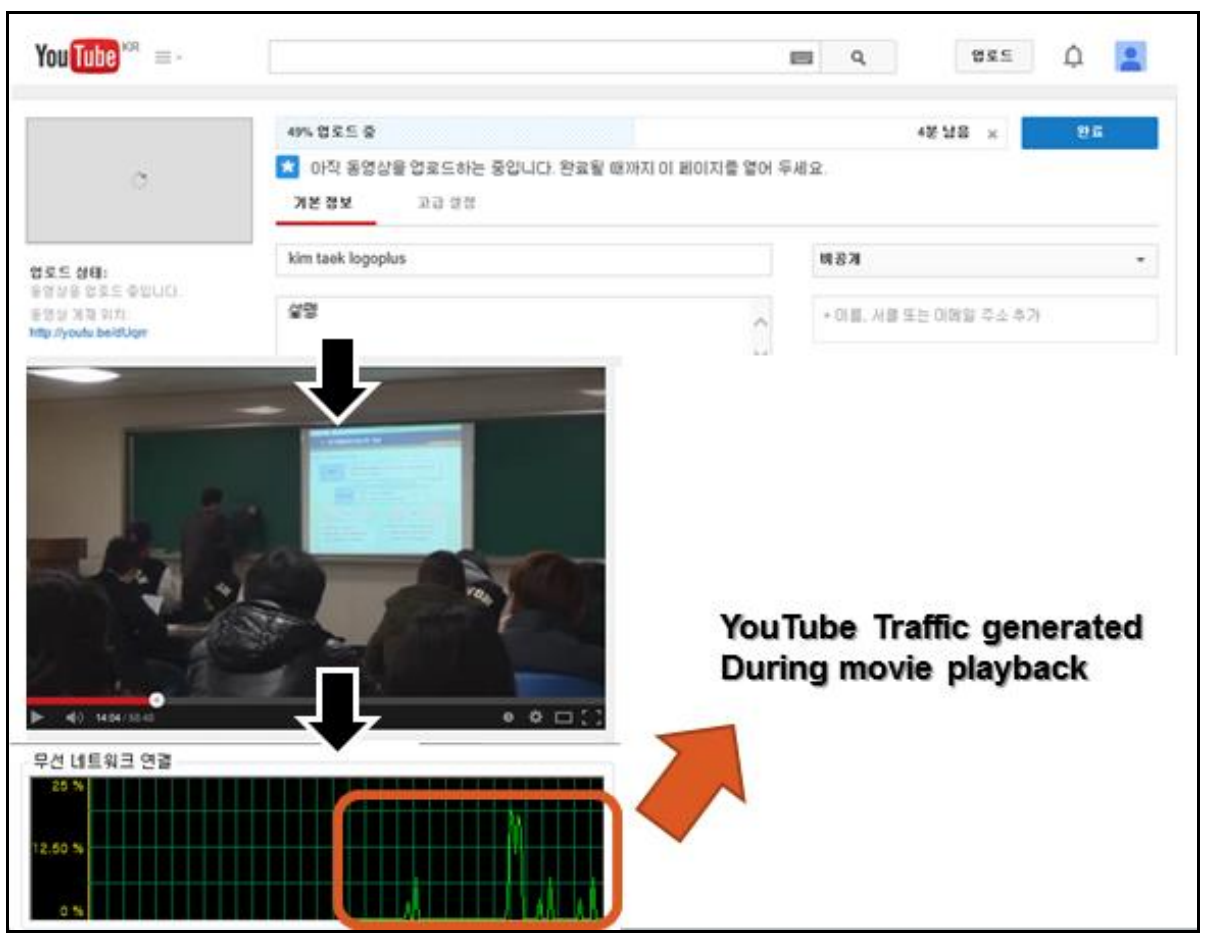

Figure 8. Playing of YouTube Video and Graph of the Traffic Data 
The video playback with YouTube helps to use efficiently the more step 3 and 4 than video service. The Google's image processing technique offers services to select the image that has been optimized for the taken image to the mobile and online services. It can reduce the burden of the operation of the learning system operators have little or no HTML knowledge.

\subsection{Optimized Online Video Selection and Traffic Analysis}

The content creation method of online classes needs several tools, but in this case we analyzed the lecture file which is produced using the digital camcorder. The recorded video was going to be converted using the "DaumPotEncoder 2.1.4.62 Beta" of "Daum Kakao Corp.". The source codec used FFMPEG decoder (WMV3 24bit), the file name, the resolution, and duration of the recorded lectures are shown in Table 3.

Table 3. Specification of a Comparison Sample Video

\begin{tabular}{c|c|c}
\hline File Name & Resolution & Duration \\
\hline Kim_lec.wmv & $1440 \times 1080$ & $00: 58: 48$ \\
\hline Yi_lec.wmv & $1280 \times 720$ & $01: 02: 20$ \\
\hline
\end{tabular}

The two video files were encoded to "WMV2 (24bit) 480X272" quality video using the video conversion program. It created a conversion to FLV method, and compared the respective data values. In this particular case of Flash Video, it may be divided into three types as follow, the FLV format that we created through the transformation is the Streaming Video method [16].

1) Embed Video: It is recognized as a graphic file in the Flash program and is used as the part of a Flash movie file.

2) Linking Video: It is an independent video as the video file to be linked, such as WMV, QuickTime Movie on the Web.

3) Streaming Video: It is a streaming media file of FLV format that is supported by the Flash MX 2004 Professional or later.

The smooth services of an online course are required, so we use the widely used Flash player which is used in teaching. We used the streaming settings shown below to create WMV and FLV stream formats.

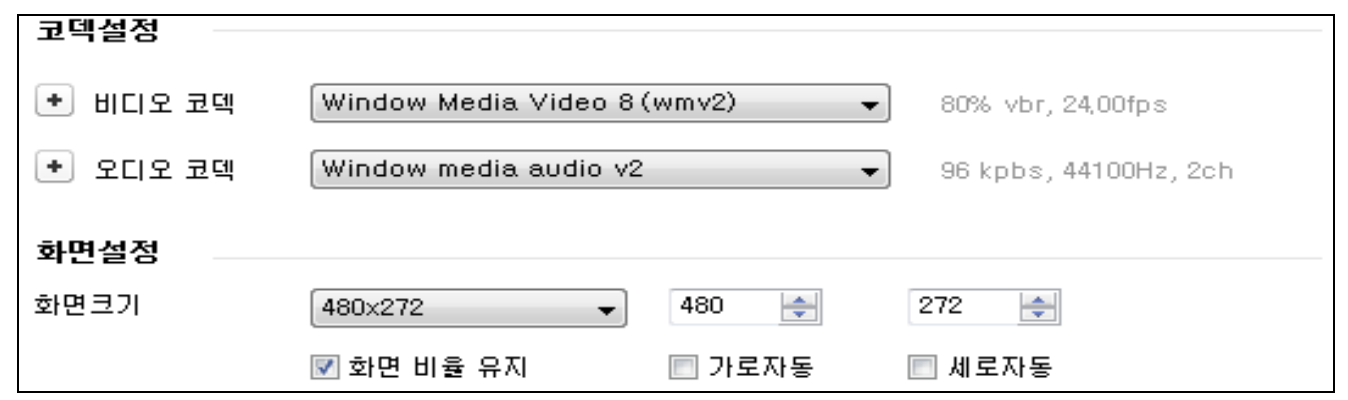

Figure 9. WMV Encoding Codec Setting Value 


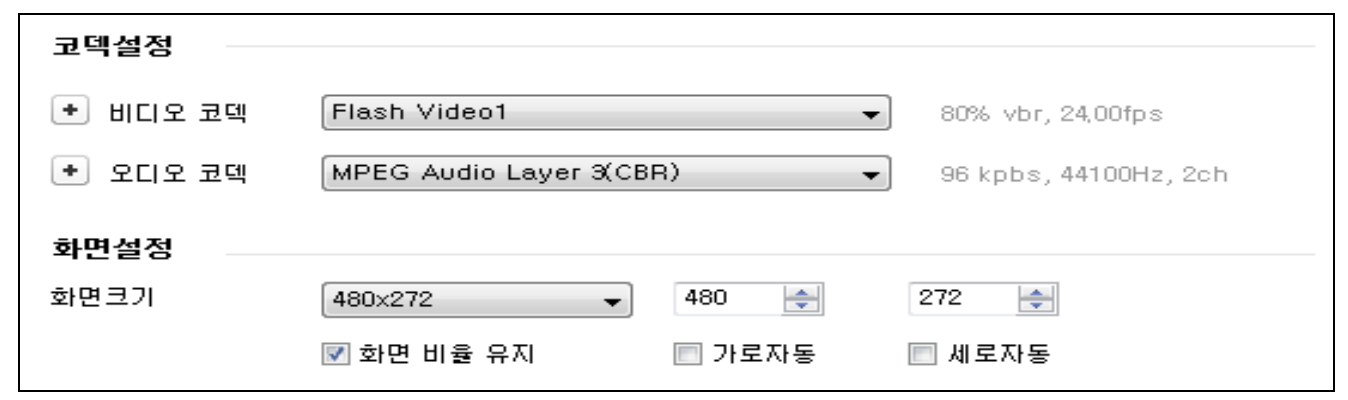

Figure 10. FLV Encoding Codec Setting Value

To analyze the result and to compare the size of the original video file with the converted video file, we divide it into the forms of WMV and FLV. The result is shown as Table 4.

Table 4. Size Comparison of Video Files

\begin{tabular}{c|c|c|c|c|c}
\hline & File Name & Duration & File Size(KB) & $\begin{array}{c}\text { CONV. } \\
\text { File Size(KB) }\end{array}$ & $\begin{array}{c}\text { Size } \\
\text { reduction(KB) }\end{array}$ \\
\hline \multirow{2}{*}{ WMV } & Kim_lec.wmv & $00: 58: 48$ & $3,623,870$ & 133,362 & $-3,490,508$ \\
\cline { 2 - 6 } & Yi_lec.wmv & $01: 02: 20$ & $2,885,457$ & 134,617 & $-2,750,840$ \\
\hline \multirow{2}{*}{ FLV } & Kim_lec.flv & $00: 58: 48$ & $3,623,870$ & 116,939 & $-3,506,931$ \\
\cline { 2 - 6 } & Yi_lec.flvv & $01: 02: 20$ & $2,885,457$ & 115,663 & $-2,769,794$ \\
\hline
\end{tabular}

When the recorded videos through a digital camcorder were converted to video lectures in a web, FLV and WMV seem to have a similar size, it suggests a relatively large reduction results that the file size of the video file conversion compared to the original video lectures.

The analysis of the quality we compared with the captured video of the transformed video is shown in Figure 11. 


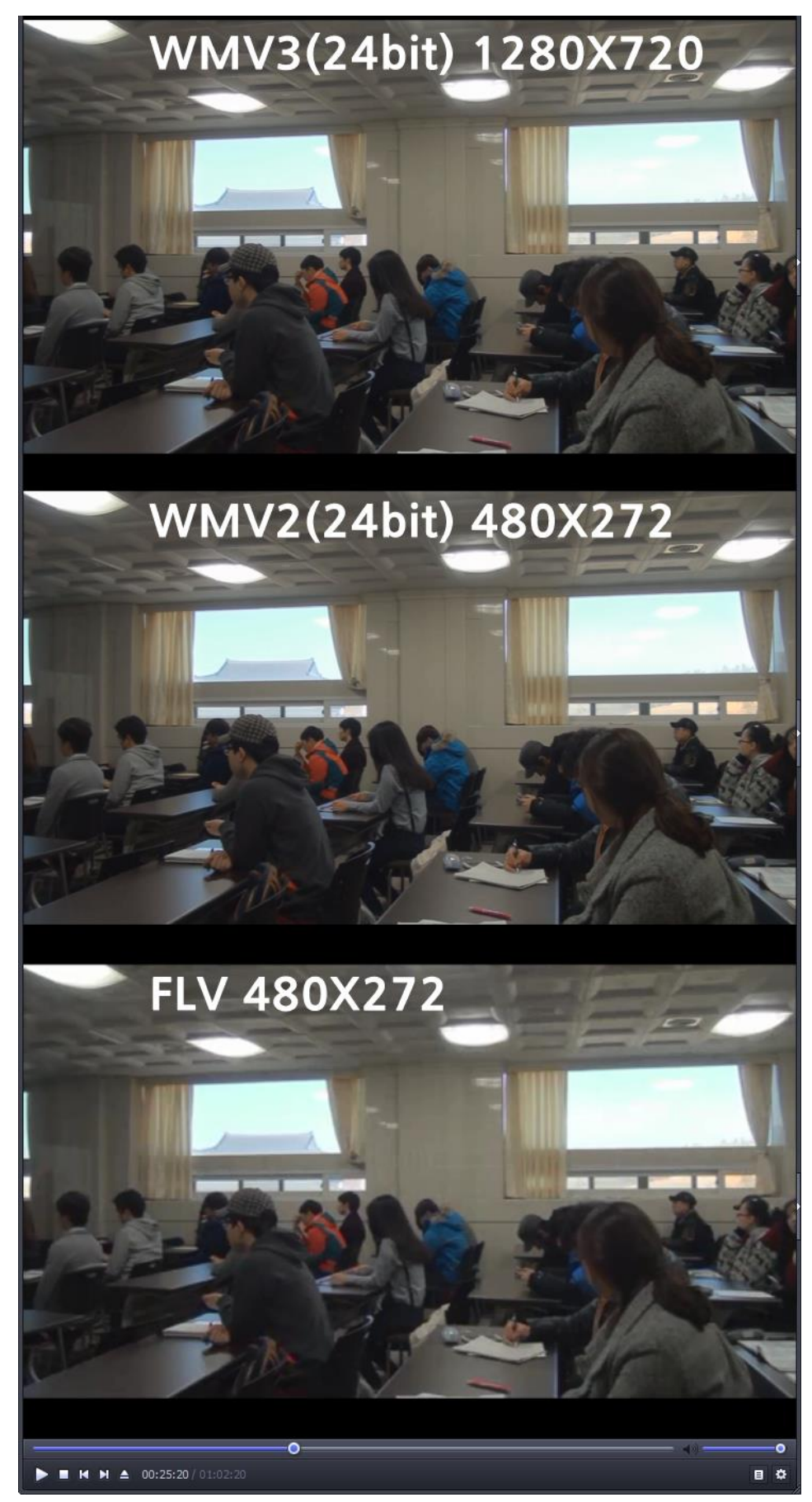

Figure 11. Comparison of the Original Video and Converted Video

When the qualities of the first original video and the converted video were compared, any big differences were not found, but the case of FLV showed fine quality impairment. However, if the quality of the online video does not make a lot of differences, it is advantageous to use the FLV file instead of WMV.

\section{The System Environment}

This implementation environment was based on CentOS 5.x, My-SQL 5.0.x, PHP 5.1.x and jQuery Mobile. This system was developed using the Gnuboard projects 
[6] that is based on GPL v2 license [5]. Figure 12 is the example of LMS that implements browser-based video upload in Figure 5. Figure 13 is account switching on mobile web with YouTube [17].

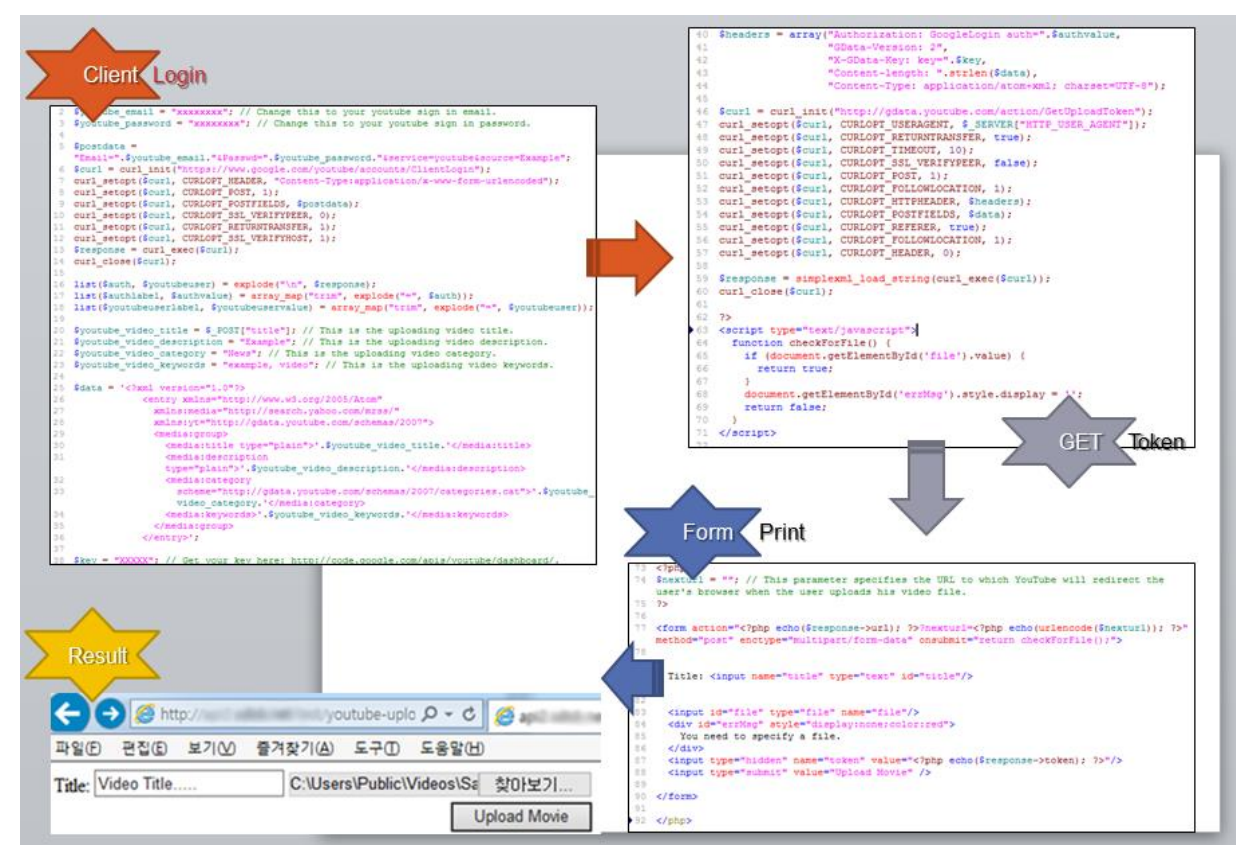

Figure 12. The Example of LMS (Video upload form)

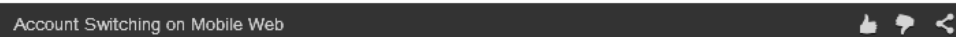

\section{- Account switching on Mobile Web}
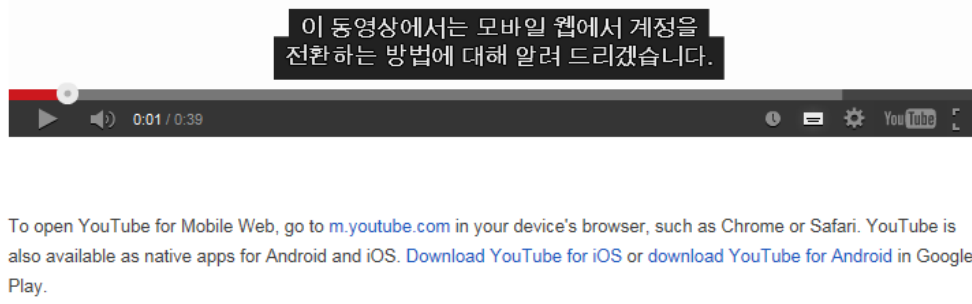

Figure 13. Account Switching on Mobile Web

\section{Conclusion}

In this paper, we propose the method of mobile learning support system based on e-learning that is reduced the phenomenon of delay due to the amount of traffic and the consumption of storage space, when you continue to play downloadable videos. This supports the low-cost and high-efficiency. 
Future research includes the segmentation of educational content between Instructors and learners, the copyright clarification, and the sharing. This feature is the part of the need to reduce the burden of sharing of content service.

\section{Acknowledgements}

This paper is a revised and expanded version of a paper entitled [Design of Small Sized Mobile Lecture Support System] presented at International Conference, Future Generation Communication and Networking 2014 held on December 20-23, 2014 at Hainan, China.

\section{Reference}

[1] C. G. Seo and S. K. Park, e-Learning System Design and Implementation for Small Sized Cyber Lecturing, Information System Review, Vol. 6, No.2, pp.161-179 (2004).

[2] Y. S. Nam, D. I. Yang and H. J. Choe, The Implementation of Interconnection Modeling between Learning Management System(LMS), The Korea Navigation Institute, Vol. 15, No. 4, pp.640-645(2011).

[3] https://developers.google.com/youtube/v3/

[4] API Overview Guide, https://developers.google.com/youtube/getting_started? hl=ko\#embedded_player.

[5] GNU General Public License v2.0, http://www.gnu.org/licenses/gpl-2.0.html.

[6] Gnuboard project, http://dev.naver.com/projects/gnuboard.

[7] ETRI, E-Learning Technology Trends, 2014 Electronics and Telecommunications Trends.

[8] KERIS, Pending Analysis of LMS on the Smart Learning, 2012 KERIS issue report research, RM 201218.

[9] J. S. Sung, Design of Collaborative Learning on Mobile Environment, IJAST Vol. 25, pp.43-54 (2010).

[10] M. F. AlAjmi, S. Khan and A. S. Zamani, Using Instructive Data Mining Methods to Revise the Impact of Virtual Classroom in E-Learning, IJAST Vol. 45, pp.125-134 (2012).

[11] J. Lee and Y. J. Lee, Development and Application of E-Learning Content for Advertising Education, IJAST Vol. 47, pp.1-12 (2012).

[12] Y. K. Cho, The Design of Learning Environment based on Facebook Social Learning, master's thesis, Ewha Womans Univ. (2011).

[13] S. J. Kim, The Development of Video Addition Information System using Open API, master's thesis, Korea Univ. (2010).

[14] G. P. Kim, The Study of Video UCC Issues and Future Research, master's thesis, Seoul National Univ. of Science \& Technology (2010).

[15] S. Lee, H. Han and Y. Jeon, Design of Small Sized Mobile Lecture Support System, Advanced Science and Technology Letters Vol.73 (FGCN 2014), pp.7-10 (2014).

[16] S. Castillo, S. Hancock, Hess, G., Using Flash MX to Create e-Learning, Rapid Intake Press, pp.86-87 (2004).

[17] Navigate YouTube on mobile browsers, https://support.google.com/youtube/answer/3008060?hl=en 